\title{
Protective effects of Notch1 signaling activation against high glucose-induced myocardial cell injury: Analysis of its mechanisms of action
}

\author{
JIAN ZHANG, BINGONG LI, ZEQI ZHENG, TING KANG, MINGHUI ZENG, YANHUA LIU and BAOHUA XIA \\ Department of Cardiology, The First Affiliated Hospital of Nanchang University, Nanchang, Jiangxi 330006, P.R. China
}

Received February 7, 2015; Accepted July 13, 2015

DOI: $10.3892 /$ ijmm.2015.2294

\begin{abstract}
Notch1 plays an important role in cardiomyocyte apoptosis and cardiac fibrosis. However, the effects of Notch1 on diabetic cardiomyopathy (DCM) and its mechanisms of action remain unclear. In the present study, we sought to investigate the role of Notch1 in, and its effects on high glucose (HG)-induced myocardial cell apoptosis and myocardial fibrosis. H9c2 cells exposed to HG were used to establish an in vitro model of myocardial injury. The $\mathrm{H} 9 \mathrm{c} 2$ cells were cultured with normal glucose (NG; $5.5 \mathrm{mmol} / \mathrm{L}-\mathrm{NG})$, and were then epxosed to $\mathrm{HG}(33 \mathrm{mmol} / \mathrm{L}-\mathrm{HG})$, a $\gamma$-secretase inhibitor (DAPT), and were transfected with a lentiviral vector containing the Notch1 intracellular domain (N1ICD; lentivirus-N1ICD). At $72 \mathrm{~h}$ following exposure to HG, DAPT or transfection with lentivirus-N1ICD, myocardial cell viability was assessed using a Cell Counting kit-8 (CCK-8) assay. Cell apoptosis was measured using Annexin V/propidium iodide (PI) double staining and flow cytometry. The mRNA expression levels of hairy/enhancer of split-1 (Hes-1) and hairy/enhancerof-split related with YRPW motif-1 (Hey-1) were measured by quantitative PCR (qPCR), while the protein expression of N1ICD, Bax, Bcl-2, transforming growth factor- $\beta 1$ (TGF- $\beta 1$ ) and connective tissue growth factor (CTGF), and the levels of phosphorylated (p-)AKT, total (t-)AKT, p-phosphoinositide 3-kinase (PI3K) and t-AKT were measured by western blot analysis. Our results revealed that exposure to $\mathrm{HG}$ induced apoptosis and upregulated TGF- $\beta 1$ and CTGF expression in the H9c 2 cardiomyocytes. Furthermore, the Notch1 and PI3K/AKT signaling pathways were activated following transfection with lentivirus-N1ICD, and this activation enhanced myocardial cell viability, prevented cardiomyocyte apoptosis and decreased TGF- $\beta 1$ and CTGF expression. On the whole, our data demonstrate that the overexpression of Notch1 prevents HG-induced
\end{abstract}

Correspondence to: Dr Bingong Li, Department of Cardiology, The First Affiliated Hospital of Nanchang University, No. 17 Yongwaizheng Street, Nanchang, Jiangxi 330006, P.R. China

E-mail: libingong08@163.com

Key words: Notch1, cardiomyocytes, apoptosis, transforming growth factor- $\beta 1$, connective tissue growth factor, PI3K/AKT cardiomyocyte apoptosis and decreases CTGF expression in $\mathrm{H} 9 \mathrm{c} 2$ cells exposed to HG. Thus, Notch1 may be used to prevent the development of DCM and to inhibit cardiac fibrosis. The findings of our study may prove to be of use in the development of novel therapeutic strategies for DCM.

\section{Introduction}

Diabetic cardiomyopathy (DCM) is a specific type of cardiomyopathy that is not associated with coronary artery disease, hypertension or other cardiac pathologies. The main pathological changes associated with DCM include extensive myocardial cell hypertrophy, apoptosis, necrosis and myocardial fibrosis (1-3). Although modern medical technology has advanced greatly in other areas, treatments for DCM remain outdated, involving the control of blood glucose levels other comprehensive measurements. Developing a more effective treatment for DCM thus remains a challenge.

Previous studies have indicated that the Notch signaling pathway plays an important role in a variety of organisms and cell types. The Notch protein is activated and cleaved by tumor necrosis factor (TNF)- $\alpha$ converting enzyme (TACE) and the $\gamma$-secretase complex, which release the Notch intracellular domain (NICD) that then binds to the transcription factor, CSL (CBF-1 in humans, suppressor of hairless in Drosophila, LAG in Caenorhabditis elegans and RBP-JК in mice) (4-6). Following the translocation of NICD into the nucleus, Notch promotes the transcription of the target genes, hairy/enhancer of split (Hes) and hairy/enhancer-of-split related with YRPW motif (Hey), which not only regulates cell proliferation, differentiation, apoptosis and adhesion, but also plays an important role in embryonic development, cancer, neurodegenerative sexual disorders, wound healing, angiogenesis and the inflammatory response $(7,8)$.

Notch1 is a member of the Notch family. In the heart, Notch1 signaling not only regulates embryonic cardiac development and differentiation, but also stimulates the proliferation of immature cardiomyocytes $(9,10)$. In addition, Notch1 signaling regulates the response to myocardial cell damage, and inhibition of Notch1 signaling can accelerate cardiac myocyte hypertrophy, degeneration and necrosis, and may eventually lead to myocardial apoptosis and fibrosis $(11,12)$. On the other hand, the inhibition of Notch1 expression can cause myocardial damage, which leads to myocardial infarction (13). At the embryonic 
stage, Notch1 signaling stimulates the proliferation of immature cardiomyocytes, and after birth, the protein expression of Notch1 gradually decreases in myocardialcells (10). Studies have indicated that Notch1 and phosphoinositide 3-kinase (PI3K)/AKT are able to interact and are dependent on phosphatase and tensin homolog deleted on chromosome 10 (PTEN) (14). In the present study, we induced Notch1 signaling by transfecting cardiomyocytes with a lentiviral vector containing Notch1 intracellular domain (N1ICD) in ain aim to protect the cells against high glucose (HG)-induced damage. The HG-induced cardiomyocyte damage was more severe when Notch1 signaling was inhibited using DAPT. To date, the effects of Notch1 signaling in DCM have not been fully determined. It is in the interest of the research community to elucidate whether the regulation of Notch1 signaling has a protective effect on myocardial cells.

Based on the pathogenesis and pathological changes associated with DCM, in this study, we performed experiments using $\mathrm{H} 9 \mathrm{c} 2$ cells exposed to HG to create a model of myocardial injury in order to investigate the role of Notch1 in, and its effects on HG-induced cardiomyocyte proliferation, apoptosis and myocardial fibrosis.

\section{Materials and methods}

Cell culture. H9c2 cardiomyocytes were purchased from the Cell Resource Center, Shanghai Institutes for Biological Sciences, Chinese Academy of Sciences (Shanghai, China). The H9c2 cells were cultured in culture plates with DMEM supplemented $10 \%$ heat-inactivated fetal bovine serum (FBS). The mixture was placed in a humidified incubator filled with $5 \% \mathrm{CO}_{2}$ at $37^{\circ} \mathrm{C}$. The cell nutrition medium was changed every 2-3 days, and the cells were subcultured once they covered $85-90 \%$ of the bottom of the culture plates. The cells were cultured under normoxic conditions with normal glucose (NG; $5.5 \mathrm{mmol} / \mathrm{L}-\mathrm{NG}$ ) for 2-3 days, before they were divided into 6 groups as folllows: i) the control group: H9c2 cells were cultured under normoxic conditions for $72 \mathrm{~h}$, without any treatment; ii) the mannitol group: H9c2 cells were cultured in mannitol (33 mmol/1; Genechem Co., Ltd., Shanghai, China) for $72 \mathrm{~h}$ (mannitol was added to rule out the effect of osmolarity in cell culture studies using high glucose); iii) the HG group: H9c2 cells were cultured in HG (33 mmol/L-HG) for $72 \mathrm{~h}$; iv) the DAPT group: H9c2 cells were cultured in HG for $24 \mathrm{~h}$, followed by the addition of DAPT $(50 \mu \mathrm{mol} / \mathrm{l}$; Shanghai Genechem Co., Ltd.) and culture for a further $48 \mathrm{~h}$; v) the Notch1 group: H9c2 cells were transfected with the lentiviral vector containing N1ICD (lentivirus-N1ICD; Shanghai Genechem Co., Ltd.) for $8 \mathrm{~h}$ and were then cultured in HG medium for a further $72 \mathrm{~h}$; and vi) the mock group: $\mathrm{H} 9 \mathrm{c} 2$ cells were transfected with an empty lentiviral vector for $8 \mathrm{~h}$ and were then cultured in HG medium for a further $72 \mathrm{~h}$. The resulting cells from each group were used in the following experiments.

Assessment of cell viability. The above-mentioned H9c2 cells were incubated in 96-well plates with 3,000 cells in each well, and this was followed by Cell Counting kit-8 (CCK-8) assay (CCK-8 solution, $10 \mu \mathrm{l} /$ well). The absorbance at $450 \mathrm{~nm}$ was measured using a Multiscan microplate reader at 1, 2, 3 and $4 \mathrm{~h}$. The cell viability for the control group was set at $100 \%$, while the viability for the other groups was expressed as a percentage of the control group.

Measurement of cell apoptosis. The H9c2 cells were incubated in 6-well plates. The cells were digested and the supernatant was transferred to a 1-ml Eppendorf tube and centrifuged at $1,000 \mathrm{rpm}$ at $4^{\circ} \mathrm{C}$ for $5 \mathrm{~min}$. The resultant supernatant was then discarded, and the precipitates were re-suspended in $500 \mu \mathrm{l}$ sterile phosphate-buffered saline (PBS). The suspension was centrifuged twice at $1,000 \mathrm{rpm}$ at $4^{\circ} \mathrm{C}$ for $5 \mathrm{~min}$. The final precipitates were re-suspended in $200 \mu 1$ binding buffer, followed by the addition of $10 \mu \mathrm{l}$ Annexin V-FITC and $10 \mu \mathrm{l}$ propidium iodide (PI; Nanjing KeyGen Biotech Co., Ltd., Nanjing, China). The mixture was vortexed gently in a dark environment at room temperature for $15 \mathrm{~min}$. Another $300 \mu \mathrm{l}$ binding buffer was added, and the apoptotic rate was measured by flow cytometry within $1 \mathrm{~h}$.

Quantitative PCR ( $P P C R)$. qPCR was performed using a 7500 Real-Time PCR system (Applied Biosystems, Carlsbad, CA, USA), using All-in-One qPCR mix (GeneCopoeia, Rockville, MD, USA). The qPCR reaction volume was $20 \mu \mathrm{l}$, containing $2 \mu \mathrm{l}$ of cDNA. The experiment was carried out according to the manufacturer's instructions. Relative mRNA expression was calculated using the comparative threshold cycle method, as previously described (15). Sequences for specific primers were as follows: Hes-1 forward, 5'-TCAAC ACGACACCGGATAAA-3' and reverse, 5'-TCAGCT GGCTCAGACTTTCA-3'; Hey-1 forward, 5'-GCGTTAT CTGAGCATCATTGAAGG' and reverse, 5'-CTGGGAA GCGTAGTTGTTGAGA-3'; GAPDH forward, 5'-GCAA GTTCAACGGCACAG-3' and reverse, 5'-GCCAGTA GACTCCACGACAT-3'.

Western blot analysis. The cells were lysed with protein lysate, and $50 \mathrm{mg}$ protein from each group were analyzed using 10\% SDS-PAGE under AU9 reducing conditions. The separated proteins were transferred onto a nitrocellulose membrane (Millipore, Billerica, MA, USA) and the membrane was blocked with $10 \%$ non-fat milk. The antibodies used were as follows: anti-N1ICD (Beijing Zhongshan Golden Bridge Biotechnology Co., Ltd., Beijing, China), anti-Bax, anti-B-cell lymphoma-2 (Bcl-2; both from Santa Cruz Biotechnology, Inc., Santa Cruz, CA, USA), anti-transforming growth factor- $\beta 1$ (TGF- $\beta 1)$ and anti-connective tissue growth factor (CTGF; both from Wuhan Boster Biological Engineering Co., Ltd., Wuhan, China), anti-phosphorylated (p-) PI3K, anti-total (t-)PI3K, anti-p-AKT and anti-t-AKT (all from Santa Cruz Biotechnology, Inc.) and anti- $\beta$-actin (Beijing Zhongshan Golden Bridge Biotechnology Co., Ltd.). The reaction mixtures were incubated for $12 \mathrm{~h}$ at $4^{\circ} \mathrm{C}$ and then for a further $4 \mathrm{~h}$ at room temperature in a shaker with the secondary antibodies [goat anti-rabbit or goat anti-mouse IgG (Zhongshan Goldenbridge Biotechnology Co., Beijing, China), goat anti-rabbit or goat anti-mouse IgG (Histostain-Plus kits; ZsBio)]. The resulting mixture was washed 3 times with TBST, and the blots were visualized by enhanced chemiluminescence.

Statistical analyses. All data were analyzed using SPSS 19.0 statistical software (SPSS,Inc.,Chicago, IL,USA). Experimental 
A

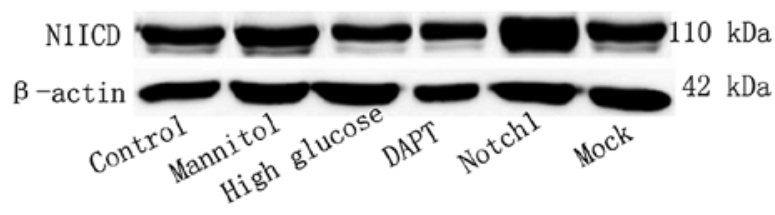

$\mathrm{C}$

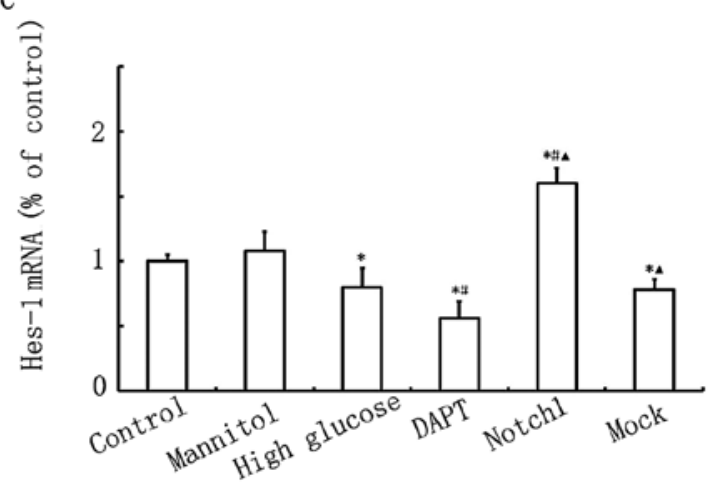

B
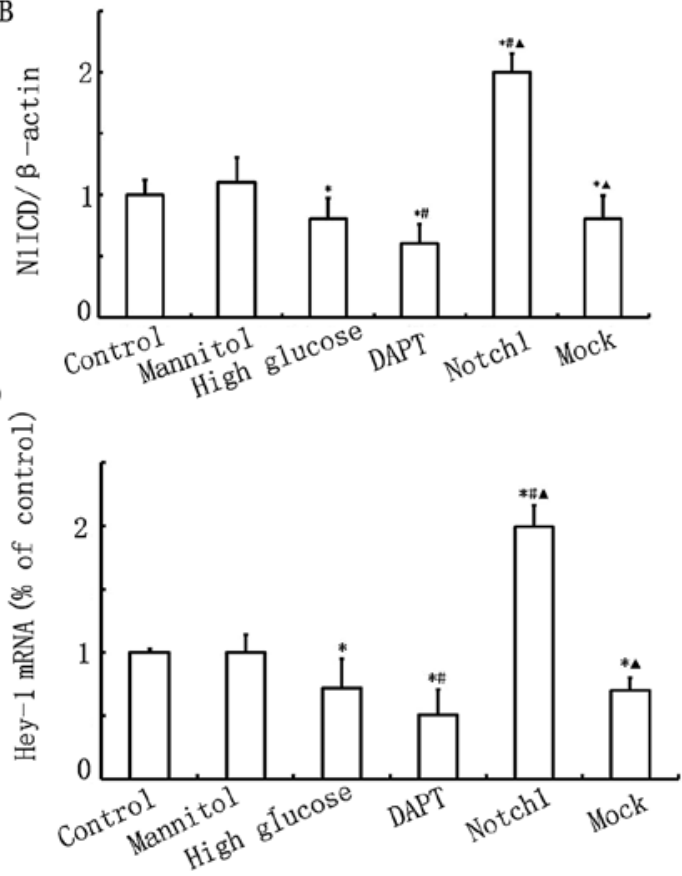

Figure 1. Notch1 signaling is activated upon high glucose (HG)-induced myocardial cell injury. (A) Western blot analysis of Notch1 intracellular domain (N1ICD) expression in the control, mannitol, HG, DAPT, Notch1 and mock groups. (B) Quantitative analysis of the N1ICD level. The mean density of N1ICD in the control group was set as $100 \%\left(\mathrm{n}=7,{ }^{*} \mathrm{P}<0.05\right.$ vs. control group, ${ }^{\#} \mathrm{P}<0.05$ vs. HG group, ${ }^{\wedge} \mathrm{P}<0.05$ vs. DAPT group). (C and D) $\mathrm{qPCR}$ of hairy/enhancer of split 1 (Hes-1) and hairy/enhancer-of-split related with YRPW motif 1 (Hey-1) mRNA expression in the control, mannitol, HG, DAPT, Notch1 and mock groups (n=7, ${ }^{*} \mathrm{P}<0.05$ vs. control group, ${ }^{*} \mathrm{P}<0.05$ vs. $\mathrm{HG}$ group, ${ }^{\wedge} \mathrm{P}<0.05$ vs. DAPT group).

data are expressed as the means $\pm \mathrm{SD}$. Comparisons between groups were compared by one-way analysis of variance (ANOVA). A value of $\mathrm{P}<0.05$ was considered to indicate a statistically significant difference.

\section{Results}

Notch1 signaling is activated upon HG-induced cardiomyocyte injury. To prove that Notch1 signaling plays an important role in HG-induced cardiomyocyte injury, we examined alterations in the expression of N1ICD and its target genes, Hes-1 and Hey-1. As shown in Fig. 1, the N1ICD, Hes-1 and Hey-1 expression levels were lower in the HG, DAPT and mock groups. The N1ICD, Hes-1 and Hey-1 expression levels were significantly higher in the Notch1 group (cells transfected with lentivirus-N1ICD prior to exposure to HG). However, in the DAPT group, the N1ICD, Hes-1 and Hey-1 expression levels were significantly decreased. Therefore, these data indicate that Notch1 signaling is activated upon HG-induced cardiomyocyte injury.

Notch1 signaling enhances $H 9 c 2$ cell viability following myocardial injury. As shown in Fig. 2, myocardial cell viability in the HG group was lower $(\mathrm{P}<0.05)$ than that in the control group. Furthermore, myocardial cell viability in the DAPT group was even lower than that in the HG group $(\mathrm{P}<0.05)$, while myocardial cell viability was significantly higher in the Notch1 group, compared with the HG group ( $\mathrm{P}<0.05$; Fig. 2). These findings indicate that the activation of the Notch1 signaling pathway enhances the viability of H9c2 cardiomyocytes exposed to HG.

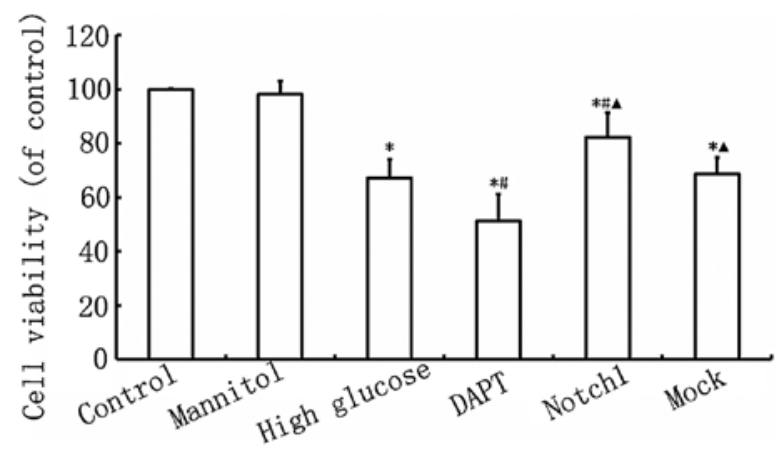

Figure 2. Effect of Notch1 signaling on the viability of H9c2 cells exposed to high glucose (HG). Cell viability was measured using a Cell Counting kit-8 (CCK-8) in the control, mannitol, HG, DAPT, Notch1 and mock groups, respectively. $\mathrm{n}=7,{ }^{*} \mathrm{P}<0.05$ vs. control group, ${ }^{\text {}} \mathrm{P}<0.05$ vs. $\mathrm{HG}$ group, ${ }^{\wedge} \mathrm{P}<0.05$ vs. DAPT group.

Notch1 signaling inhibits $H G$-induced H9c2 cell apoptosis. To confirm the protective effects of Notch1 signaling against HG-induced $\mathrm{H} 9 \mathrm{c} 2$ cell damage, cell apoptosis was determined by measuring the protein expression levels of Bax and Bcl-2 by western blot analysis, and by Annexin V-FITC/PI double staining and flow cytometry. The results from western blot analysis and flow cytometry are presented in Figs. 3 and 4. As shown in Fig. 3, the ratio of Bax to Bcl-2 in the $\mathrm{HG}$ group was higher than that in the control group $(\mathrm{P}<0.05)$. Furthermore, the ratio of Bax to Bcl-2 in the DAPT group was even higher than that in the HG group $(\mathrm{P}<0.05)$, while the ratio of Bax to Bcl-2 was significantly lower in the Notch1 group compared with that in the HG group $(\mathrm{P}<0.05)$. Our findings indicate that 

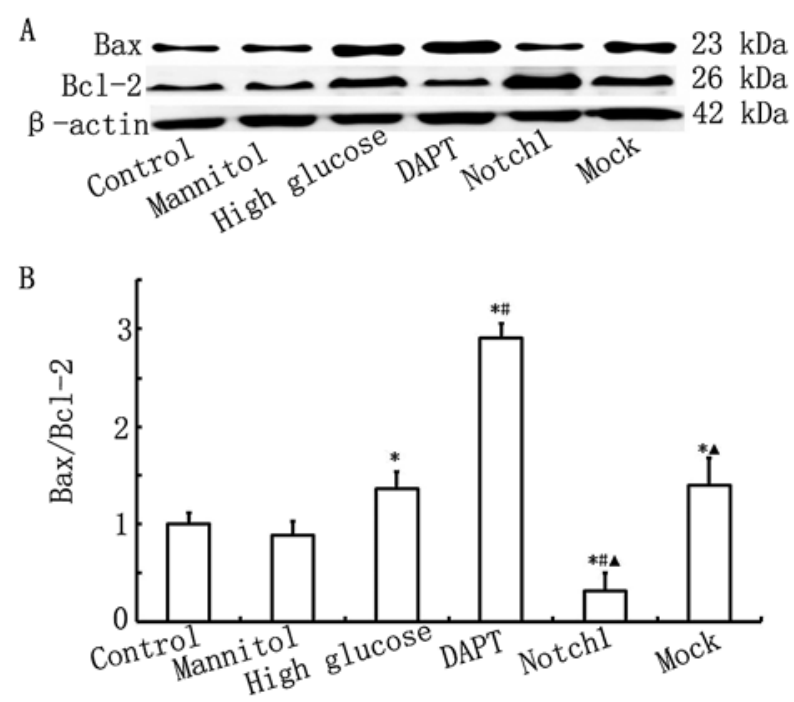

Figure 3. Effect of Notch1 signaling on HG-induced H9c2 cell apoptosis. (A) Western blot analysis of Bax and B-cell lymphoma-2 (Bcl-2) protein levels. (B) Quantitative analysis of the ratio of Bax to $\mathrm{Bcl}-2$ in the control, mannitol, HG, DAPT, Notch1 and mock groups. The ratio of mean densities for Bax to Bcl-2 in the control group was set as $100 \%$ ( $\mathrm{n}=7,{ }^{*} \mathrm{P}<0.05$ vs. control group, ${ }^{,} \mathrm{P}<0.05$ vs. HG group, ${ }^{\mathrm{P}} \mathrm{P}<0.05$ vs. DAPT group).

exposure to HG induces $\mathrm{H} 9 \mathrm{c} 2$ myocardial cell apoptosis and that this is inhibited by the activation of Notch1 signaling.

As shown in Fig. 4, the apoptotic rate in the HG group was higher than that in the control group $(\mathrm{P}<0.05)$, and the apoptotic rate in the DAPT group was higher than that in the HG group $(\mathrm{P}<0.05)$. When Notch1 signaling was activated following transfection with lentivirus-N1ICD, the apoptotic rate decreased significantly and was lower than that in the HG group $(\mathrm{P}<0.05)$. These results further confirm that the activation of the Notch1 signaling pathway inhibits HG-induced cardiomyocyte apoptosis.

Activation of Notchl signaling decreases the expression of TGF- $\beta 1$ and CTGF induced in H9c2 myocardial cells by exposure to $H G$. To further confirm the protective effects of Notch1 signaling in $\mathrm{H} 9 \mathrm{c} 2$ cells exposed to $\mathrm{HG}$, the protein expression levels of TGF- $\beta 1$ and CTGF were measured by western blot analysis. Both proteins are important factors in myocardial fibrosis (16). As shown in Fig. 5, the expression levels of TGF- $\beta 1$ and CTGF were higher $(\mathrm{P}<0.05)$ in the HG group compared with those in the control group. The protein expression levels of TGF- $\beta 1$ and CTGF were even higher $(\mathrm{P}<0.05)$ in the DAPT group compared with those in the HG group, while these levels were significantly lower $(\mathrm{P}<0.05)$ in the Notch1 group compared with those in the HG group. The above findings indicate that exposure to HG increases the expression of TGF- $\beta 1$ and CTGF in $\mathrm{H} 9 \mathrm{c} 2$ myocardial cells, and that the activation of Notch1 signaling inhibits this increase in the expression of pro-fibrotic factors.

Overexpression of Notchl activates the PI3K/AKT pathway. To determine the cardioprotective effects of the Notch1 signaling pathway in relation to HG-induced $\mathrm{H} 9 \mathrm{c} 2$ cell damage, we measured the expression levels of p-AKT/t-AKT

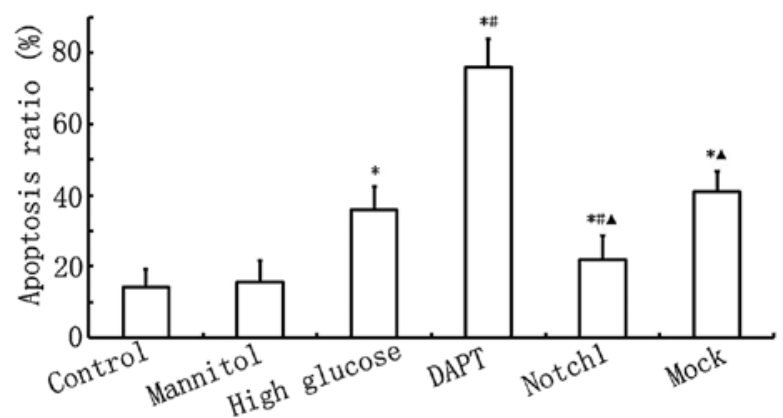

Figure 4. Effects of Notch1 signaling on HG-induced H9c2 cell apoptosis Ratio of apoptotic cells in the control, mannitol, HG, DAPT, Notch1 and mock groups. The ratio of mean densities for Bax and B-cell lymphoma-2 (Bcl-2) in the control group was set as $100 \%(n=7$ per group, ${ }^{*} \mathrm{P}<0.05$ vs. control group, ${ }^{\sharp} \mathrm{P}<0.05$ vs. HG group, ${ }^{\wedge} \mathrm{P}<0.05$ vs. DAPT group).

and p-PI3K/t-PI3K. As shown in Fig. 6, no significant differences were observed in the levels of t-AKT between the different groups. However, AKT activity was lower in the HG, DAPT and mock groups, as indicated by the downregulation in the levels of p-AKT, which were lower in the DAPT group but higher in the Notch1 group. As shown in Fig. 6, the levels of p-PI3K were lower in the HG, DAPT and mock groups, and were particularly lowe in the DAPT group, whereas in the Notch1 group, the level of p-PI3K was significantly higher. These data suggest that the activation of Notch1 signaling in turn activates the PI3K/AKT pathway. Our data also suggest that the acvitaion of Notch1 signaling exerts cardioprotective effects, possibly through cross-talk with the PI3K/AKT signaling pathway.

\section{Discussion}

Our study demonstrated that HG-induced cardiomyocyte apoptosis led to an increase in TGF- $\beta 1$ and CTGF expression in the $\mathrm{H} 9 \mathrm{c} 2$ cells. Our findings also indicated that the overexpression of Notch1 prevented HG-induced cardiomyocyte apoptosis and decreased CTGF expression in the H9c2 cells exposed to HG; thus, Notch1 plays a protective role and may reduce the severity of cardiac fibrosis.

Despite the fact that the Notch1 signaling pathway plays an important role in cardiovascular disease, to the best of our knowledge, prior to this study, there was no in-depth report on the role of Notch1 signaling in DCM. Our findings demonstrated that the overexpression of Notch1 exerted therapeutic effects against HG-induced H9c2 cardiomyocyte injury. Our data suggest that Notch1 lentiviral gene transduction into myocardial cells is feasible and beneficial, and that it is possible to develop novel Notch1-dependent therapeutic strategies for DCM.

Previous studies have confirmed that there is a complex association between Notch1 signaling and the PI3K/AKT pathway, and that the effects of the cross-talk between these pathways are also quite complex (17-20). Our study demonstrated that following the transfection of H9c2 cardiomyocytes with lentivirus-N1ICD, the expression of Notch1 increased and this led to the activation of the PI3K/AKT pathway. It has been demonstrated that the Notch1 targt gene, Hes-1, activates 
A

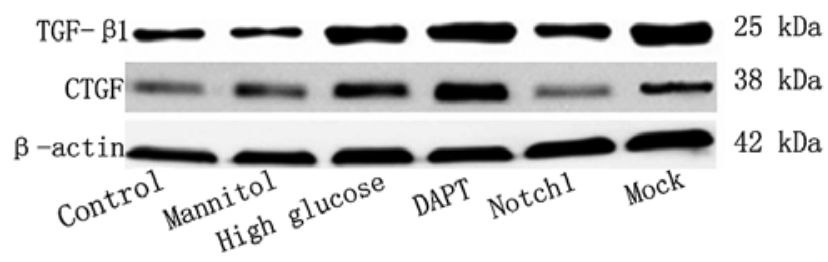

B

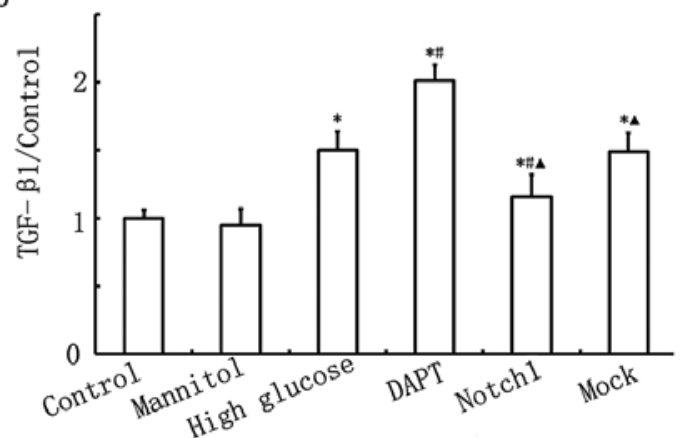

$\mathrm{C}$

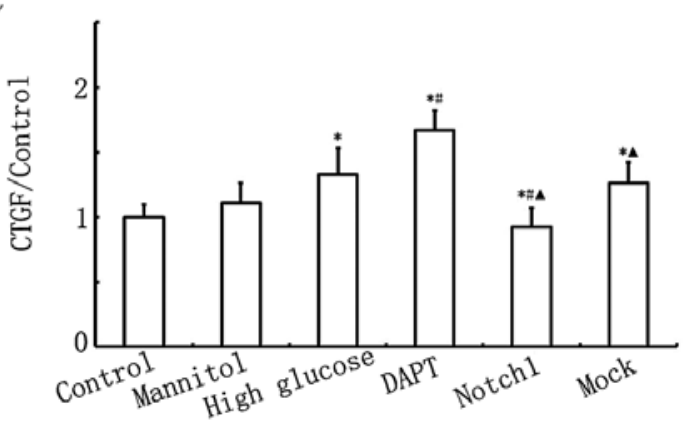

Figure 5. Effects of Notch1 signaling on high glucose (HG)-induced H9c2 cell fibrosis. (A) Western blot analysis of transforming growth factor- $\beta 1$ (TGF- $\beta 1$ ) and connective tissue growth factor (CTGF) protein levels. (B and C) Quantitative analysis of TGF- $\beta 1$ and CTGF in the control, mannitol, HG, DAPT, Notch1 and mock groups. The mean densities of TGF- $\beta 1$ and CTGF in the control group were set as $100 \%\left(\mathrm{n}=7,{ }^{*} \mathrm{P}<0.05\right.$ vs. control group, ${ }^{\#} \mathrm{P}<0.05$ vs. HG group, $\triangle \mathrm{P}<0.05$ vs. DAPT group).

A

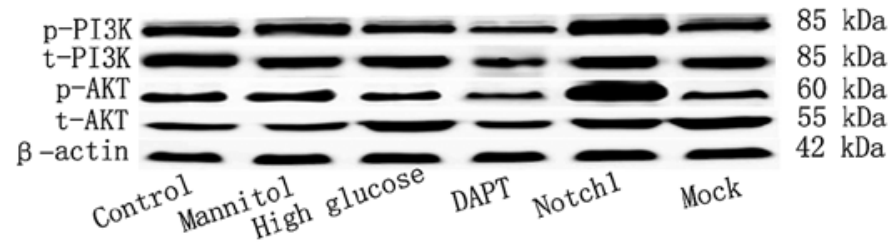

B

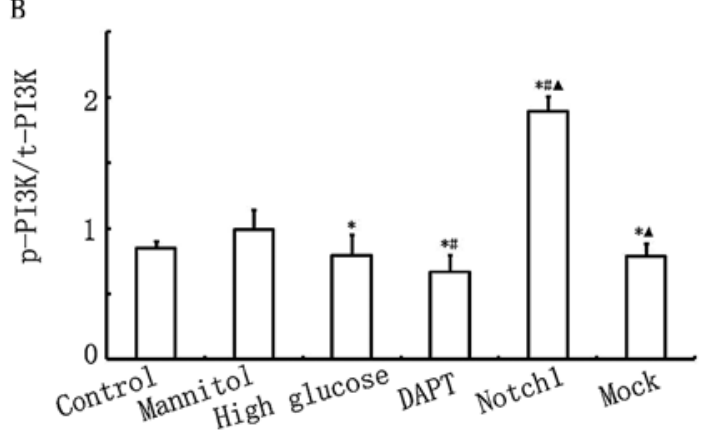

$\mathrm{C}$

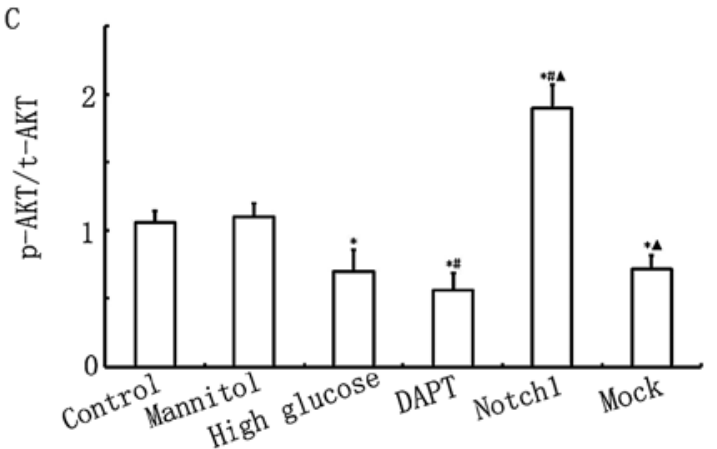

Figure 6. Notch1 signaling cross-talks with the phosphoinositide 3-kinase (PI3K)/AKT signaling pathway. (A) Western blot analysis of phosphorylated (p)-PI3K/ total (t)-PI3K and p-AKT t-AKT protein levels. (B and C) Quantitative analysis of the ratio of p-PI3K to t-PI3K and the ratio of p-AKT to t-AKT in the control, mannitol, high glucose (HG), DAPT, Notch1 and mock groups. The ratio of mean densities for p-PI3K to t-PI3K and the ratio of p-AKT to t-AKT in the control group was set as $100 \%\left(\mathrm{n}=7,{ }^{,} \mathrm{P}<0.05\right.$ vs. control group, ${ }^{\not} \mathrm{P}<0.05$ vs. $\mathrm{HG}$ group, ${ }^{\wedge} \mathrm{P}<0.05$ vs. DAPT group).

the PI3K/AKT pathway by negatively regulating PTEN. This downregulation is achieved mainly through the upregulation of receptor tyrosine kinases (RTKs) and the downregulation of PTEN. PI3K is phosphorylated, while p-PI3K simultaneously gathers and phosphorylates AKT (21). p-AKT further regulates downstream apoptotic factors, such as the Bcl-2 family, caspase-9 and -3, and X-linked inhibitor of apoptosis protein (XIAP). Among them, the AKT dependence of the Bcl-2 family is critical during the pathogenesis of apoptosis (22-24).
Myocardial interstitial fibrosis is a key risk factor in the progression of DCM. Cardiac fibroblast proliferation and activation lead to the secretion of a large amount of collagen types I and III, resulting in the accumulation and imbalance of those collagens in myocardial tissue (11). TGF- $\beta 1$ has been the focus of recent research and has been proven to be a regulatory element in many tissues and cell fibrosis $(25,26)$. It has been reported that TGF- $\beta 1$ signal transduction plays an important role in a number of fibrotic diseases by activating Smad 
protein $(27,28)$. In addition, TGF- $\beta 1$ is thought to be involved in the transformation of fibroblasts by upregulating the expression of TGF- $\beta 1$ and CTGF in rat pulmonary fibrosis (26). Activated TGF- $\beta 1$ contributes to the pathogenesis of the fibrotic interstitium observed in DCM. Furthermore, HG enhances the activity of the transcriptional co-activator, p300, leading to the activation of TGF- $\beta$ via the acetylation of Smad2 (29).

During myocardial injury, several signaling pathways function together and affect each other. The TGF- $\beta 1$ pathway activates the Notch1 signaling pathway, while the activated Notch1 signaling pathway inhibits the TGF- $\beta 1$ pathway. Sassoli et al (30) reported that the hormone relaxin (RLX) downregulated the expression of TGF- $\beta 1$ and type I collagen by activating the Notch1 signaling pathway, which further inhibited TGF- $\beta 1$-mediated fibroblast myofibroblast transition. However, the inhibitory effects of RLX decreased significantly following intervention with the $\gamma$-secretase inhibitor, DAPT. Furthermore, it has been suggested that the activation of the PI3K/AKT pathway directly inhibits the expression of TGF- $\beta 1$, and thus inhibits myocardial fibrosis $(31,32)$.

The present study confirmed that TGF- $\beta 1$ and CTGF are both expressed in $\mathrm{H} 9 \mathrm{c} 2$ cells. The epxosure of $\mathrm{H} 9 \mathrm{c} 2$ cells to HG led to the upregulation of the expression of pro-fibrotic factors, which may result in cardiac fibrosis. Our findings further demonstrated that the overexpression of Notch1 in H9c2 cells led to lower expression levels of CTGF, which were induced by TGF- $\beta 1$. These results suggest that Notch1 is involved in cardiac remodeling, acting as a fibrogenic mediator of TGF- $\beta 1$ and affecting the cardiac myoblasts. We hypothesized that the mechanism responsible for this is that the overexpression of Notch1 activated the PI3K/AKT pathway, which further inhibited the expression of TGF- $\beta 1$ and eventually downregulated the expression of CTGF.

In recent years, the Notch1 signaling pathway has attracted widespread attention (10-15,17-20,33). However, previous studies in this field have mainly focused on the cellular $(10,12,20)$ and animal levels $(11,19,33)$ and no research has been carried out at the human clinical trial level. In our study, human H9c2 cells from were used to create a model of myocardial injury. Using this model, we investigated protective effects of Notch1 against myocardial injury as well as its mechanisms of action. That being said, as our study was carried out at the in vitro level and the in vivo environment is more complex, further studies are warranted to determine whether Notch1 would have the same protective effect in vitro.

In conclusion, this study explored the protective effects of Notch1 signaling against HG-induced myocardial injury in vitro, as well as its mechanism of action, and laid the foundation for future in vitro experiments. Our data may aid the development of novel treatment strategies for DCM. As the processes of myocardial apoptosis and fibrosis are regulated by a complex system of cytokines and signaling pathways, further clinical studies are required to confirm the safety and efficacy of any new treatment strategy for DCM.

\section{Acknowledgements}

This study was supported by the National Natural Science Foundation of China (81041097) and the Natural Science Foundation of Jiangxi Province (20142BAB205040). We thank
Dr Wan Zhang and Dr Junyi Zeng for expert technical assistance and critical comments on the manuscript.

\section{References}

1. Fiordaliso F, De Angelis N, Bai A, Cuccovillo I, Salio M, Serra DM, Bianchi R, Razzetti R, Latini R and Masson S: Effect of beta-adrenergic and renin-angiotensin system blockade on myocyte apoptosis and oxidative stress in diabetic hypertensive rats. Life Sci 81: 951-959, 2007

2. Bell DS: Diabetic cardiomyopathy. Diabetes Care 26: 2949-2951, 2003.

3. Wen HL, Liang ZS, Zhang R and Yang K: Anti-inflammatory effects of triptolide improve left ventricular function in a rat model of diabetic cardiomyopathy. Cardiovasc Diabetol 12: 50, 2013.

4. Leong KG and Karsan A: Recent insights into the role of Notch signaling in tumorigenesis. Blood 107: 2223-2233, 2006.

5. Ji X, Wang Z, Geamanu A, Sarkar FH and Gupta SV: Inhibition of cell growth and induction of apoptosis in non-small cell lung cancer cells by delta-tocotrienol is associated with notch-1 down-regulation. J Cell Biochem 112: 2773-2783, 2011.

6. Cook KM and Figg WD: Angiogenesis inhibitors: current strategies and future prospects. CA Cancer J Clin 60: 222-243, 2010.

7. Lefort K and Dotto GP: Notch signaling in the integrated control of keratinocyte growth/differentiation and tumor suppression. Semin Cancer Biol 14: 374-386, 2004.

8. Sjölund J, Manetopoulos C, Stockhausen MT and Axelson H: The Notch pathway in cancer: differentiation gone awry. Eur J Cancer 41: 2620-2629, 2005.

9. MacGrogan D, Nus M and de la Pompa JL: Notch signaling in cardiac development and disease. Curr Top Dev Biol 92: 333-365, 2010.

10. Collesi C, Zentilin L, Sinagra G and Giacca M: Notch1 signaling stimulates proliferation of immature cardiomyocytes. J Cell Biol 183: 117-128, 2008

11. Nemir M, Metrich M, Plaisance I, Lepore M, Cruchet S, Berthonneche C, Sarre A, Radtke F and Pedrazzini T: The Notch pathway controls fibrotic and regenerative repair in the adult heart. Eur Heart J 269: 715-727, 2012.

12. Zhou XL, Wan L and Liu JC: Activated Notch1 reduces myocardial ischemia reperfusion injury in vitro during ischemic postconditioning by crosstalk with the RISK signaling pathway. Chin Med J (Engl) 126: 4545-4551, 2013.

13. Li Y, Hiroi Y and Liao JK: Notch signaling as an important mediator of cardiac repair and regeneration after myocardial infarction. Trends Cardiovasc Med 20: 228-231, 2010.

14. Gutierrez A and Look AT: NOTCH and PI3K-AKT pathways intertwined. Cancer Cell 12: 411-413, 2007.

15. Li Y, Li B, Zhang C, Zhang J, Zeng M and Zheng Z: Effect of NRG-1/ErbB signaling intervention on the differentiation of bone marrow stromal cells into sinus node-like cells. J Cardiovasc Pharmacol 63: 434-440, 2014.

16. Matsumoto Y, Niimi N and Kohyama K: Characterization of fibrosis-promoting factors and siRNA-mediated therapies in C-protein-induced experimental autoimmune myocarditis. Cell Immunol 279: 70-77, 2012.

17. Wang H, Cheng H, Shao Q, Dong Z, Xie Q, Zhao L, Wang Q, Kong $\mathrm{B}$ and Qu X: Leptin-promoted human extravillous trophoblast invasion is MMP14 dependent and requires the cross talk between Notch1 and PI3K/Akt signaling. Biol Reprod 90: 78-86, 2014.

18. Xiao W, Chen X and He M: Inhibition of the Jagged/Notch pathway inhibits retinoblastoma cell proliferation via suppressing the PI3K/Akt, Src, p38MAPK and Wnt/ $\beta$ catenin signaling pathways. Mol Med Rep 10: 453-458, 2014.

19. Gude NA, Emmanuel G, Wu W, Cottage CT, Fischer K, Quijada P, Muraski JA, Alvarez R, Rubio M, Schaefer E and Sussman MA: Activation of Notch-mediated protective signaling in the myocardium. Circ Res 102: 1025-1035, 2008.

20. Wang XM, Yao M, Liu SX, Hao J, Liu QJ and Gao F: Interplay between the Notch and PI3K/Akt pathways in high glucose-induced podocyte apoptosis. Am J Physiol Renal Physiol 306: F205-F213, 2014.

21. Xie F, Su M, Qiu W, Zhang M, Guo Z, Su B, Liu J, Li X and Zhou L: Kaempferol promotes apoptosis in human bladder cancer cells by inducing the tumor suppressor, PTEN. Int J Mol Sci 14: 21215-21226, 2013. 
22. Katare RG, Kakinuma Y, Arikawa M, Yamasaki F and Sato T: Chronic intermittent fasting improves the survival following large myocardial ischemia by activation of BDNF/VEGF/PI3K signaling pathway. J Mol Cell Cardiol 46: 405-412, 2009.

23. Tiwari RV, Parajuli P and Sylvester PW: $\gamma$-Tocotrienol-induced autophagy in malignant mammary cancer cells. Exp Biol Med (Maywood) 239: 33-44, 2014.

24. Chang TH, Liu XY, Zhang XH and Wang HL: Effects of dl-praeruptorin A on interleukin-6 level and Fas, bax, bcl-2 protein expression in ischemia-reperfusion myocardium. Acta Pharmacol Sin 23: 769-774, 2002

25. Yi X, Li X, Zhou Y, Ren S, Wan W, Feng G and Jiang X: Hepatocyte growth factor regulates the TGF- $\beta 1$-induced proliferation, differentiation and secretory function of cardiac fibroblasts. Int J Mol Med 34: 381-390, 2014.

26. Zhang L, Li Y, Liang C and Yang W: CCN5 overexpression inhibits profibrotic phenotypes via the PI3K/Akt signaling pathway in lung fibroblasts isolated from patients with idiopathic pulmonary fibrosis and in an in vivo model of lung fibrosis. Int J Mol Med 33: 478-486, 2014.

27. Wang M, Zhao D, Spinetti G, Zhang J, Jiang LQ, Pintus G, Monticone R and Lakatta EG: Matrix metalloproteinase 2 activation of transforming growth factor- $\beta 1$ (TGF- $\beta 1$ ) and TGF- $\beta 1$-type II receptor signaling within the aged arterial wall. Arterioscler Thromb Vasc Biol 26: 1503-1509, 2006.
28. Beaumont J, López B, Hermida N, Schroen B, San José G, Heymans S, Valencia F, Gómez-Doblas JJ, De Teresa E, Díez J and González A: microRNA-122 down-regulation may play a role in severe myocardial fibrosis in human aortic stenosis through TGF- $\beta 1$ up-regulation. Clin Sci (Lond) 126: 497-506, 2014.

29. Bugyei-Twum A, Advani A, Advani SL, Zhang Y, Thai K, Kelly DJ and Connelly KA: High glucose induces Smad activation via the transcriptional coregulator p300 and contributes to cardiac fibrosis and hypertrophy. Cardiovasc Diabetol 13: 89, 2014.

30. Sassoli C, Chellini F, Pini A, Tani A, Nistri S, Nosi D, ZecchiOrlandini S, Bani D and Formigli L: Relaxin prevents cardiac fibroblast-myofibroblast transition via notch-1-mediated inhibition of TGF- $\beta /$ Smad3 signaling. PLoS One 8: e63896, 2013.

31. Voloshenyuk TG, Landesman ES, Khoutorova E, Hart AD and Gardner JD: Induction of cardiac fibroblast lysyl oxidase by TGF- $\beta 1$ requires PI3K/Akt, Smad3, and MAPK signaling. Cytokine 55: 90-97, 2011.

32. Chung EJ, Sohn YH, Kwon SH, Jung SA and Lee JH: Lithium chloride inhibits TGF- $\beta 1$-induced myofibroblast transdifferentiation via PI3K/Akt pathway in cultured fibroblasts from Tenon's capsule of the human eye. Biotechnol Lett 36: 1217-1224, 2014.

33. Liu J, Dong F, Jeong J, Masuda T and Lobe CG: Constitutively active Notch 1 signaling promotes endothelial-mesenchymal transition in a conditional transgenic mouse model. Int J Mol Med 34: 669-676, 2014. 